\title{
Relative Property (T) for Topological Groups
}

\author{
Jicheng Tao1, Wen Yan' \\ ${ }^{1}$ Department of Mathematics, China Jiliang University, Hangzhou, China \\ ${ }^{2}$ Department of Mathematics, Tuskegee University, Tuskegee, USA \\ Email: taojc@cjlu.edu.cn, wenyanmath@gmail.com
}

Received 18 August 2014; revised 16 September 2014; accepted 6 October 2014

Copyright (C) 2014 by authors and Scientific Research Publishing Inc.

This work is licensed under the Creative Commons Attribution International License (CC BY).

http://creativecommons.org/licenses/by/4.0/

(c) (i) Open Access

\section{Abstract}

In this paper, we investigate Kazhdan's relative Property $(\mathrm{T})$ for pairs $(G, X)$, where $G$ is a topological group and $X$ is any subset of $G$. We show that the pair $(G, X)$ has Property (FH) and every function conditionally of negative type on $G$ is $X$-bounded if the pair $(G, X)$ has relative Property (T). We also prove that $G$ has Property (T) when $G$ is a $\sigma$-compact locally compact group generated by its subgroups $H_{1}, \cdots, H_{n}$ and the pair $\left(G, H_{i}\right)$ has relative Property (T) for all $i$.

\section{Keywords}

Relative Property (T) of Topological Group, Function Conditionally of Negative Type, Property (FH)

\section{Introduction}

In the mid 60's, Kazhdan defined the following Property (T) for locally compact groups and used this to prove that a large class of lattices are finitely generated.

Definition 1.1. [1] A topological group $G$ has Property (T) if there exist a compact subset $Q$ and a real number $\varepsilon>0$ such that, whenever $\pi$ is a continuous unitary representation of $G$ on a Hilbert space $H$ for which there exists a vector $\xi \in H$ of norm 1 with $\sup _{q \in Q}\|\pi(q) \xi-\xi\|<\varepsilon$, then there exists an invariant vector, namely a vector $\eta \neq 0$ in $H$ such that $\pi(g) \eta=\eta$ for all $g \in G$.

Since then, Property (T) has been studied extensively and there are a lot of publications. One can see [2]-[5].

The notion of relative Property (T) for a pair $(G, N)$, where $N$ is a normal subgroup of $G$, was implicit in 
Kazhdan's paper [5], and later made explicit by Margulis [6]. The definition of relative Property (T) has been extended in [7] to pairs $(G, H)$ with $H$ not necessarily normal in $G$. In order to obtain more information about the unitary dual of locally compact group, Cornulier in [3] extended the definition of relative Property (T) to pairs $(G, X)$, where $X$ is any subset of the locally compact group $G$.

We know that if the pair $(G, X)$ has relative Property (T), then there exists an open, compactly generated subgroup $H$ of $G$, containing $X$, such that $(H, X)$ has relative Property (T). Shalom [8] generalizes Kazhdan's definition of Property (T) to topological groups that are not locally compact. There are many natural examples such as the loop group of all continuous functions from a circle to $S L_{n}(C), n \geq 3$ and the pair $\left(S L_{3}(Z) \times Q, \Gamma \times\{0\}\right) \quad\left(\Gamma\right.$ is a subgroup of $S L_{3}(Z)$ ), which both have Property $(\mathrm{T})$.

Inspired by the work of Cornulier and Shalom, in this paper, we go further in this direction and try to extend Cornulier's result from locally compact groups to topological groups. The motivation for this is that, given a topological group $G$, the knowledge of the family of subsets $X$ such that has relative Property (T) contains much more precise information than the bare information whether $G$ has Property (T). On the other hand, relative Property (T) for topological groups is very important to discuss Haagerup Property.

\section{Preliminaries}

We first introduce some of basic notations and terminologies, the details can be found in [1].

Definition 2.1. [1] Let $G$ be a topological group, and $H$ be a closed subgroup. The pair $(G, H)$ has Property (T) if there exist a compact subset $Q$ and a real number $\varepsilon>0$ such that, whenever $\pi$ is a continuous unitary representation of $G$ on a Hilbert space $\hbar$ for which there exists a vector $\xi \in \hbar$ of norm 1 with $\sup _{q \in Q}\|\pi(q) \xi-\xi\|<\varepsilon$, then there exists an invariant vector, namely a vector $\eta \neq 0$ in $\hbar$ such that $\pi(g) \eta=\eta$ for all $g \in H$.

Definition 2.2. Let $\pi$ be an orthogonal representation of the topological group $G$ on a real Hilbert space $H^{0}$.

1) A continuous mapping $b: G \rightarrow H^{0}$ such that $b(g h)=b(g)+\pi(g) b(h)$, for all $g, h \in G$ is called a 1cocycle with respect to $\pi$.

2) A 1-cocycle $b: G \rightarrow H^{0}$ for which there exists $\xi \in H^{0}$ such that $b(g)=\pi(g) \xi-\xi$, for all $g \in G$ is called a 1-coboudary with respect to $\pi$.

3) The space $Z^{1}(G, \pi)$ of all 1-cocycles with respect to $\pi$ is a real vector space under the pointwise operations, and the set $B^{1}(G, \pi)$ of all 1-coboundaries is a subspace of $Z^{1}(G, \pi)$. The quotient vector space $H^{1}(G, \pi)=Z^{1}(G, \pi) / B^{1}(G, \pi)$ is called the first cohomology group with coefficients in $\pi$.

4) Let $b \in Z^{1}(G, \pi)$. The affine isometric action associated to a cocycle $b \in Z^{1}(G, \pi)$ is the affine isometric action $\alpha$ of $G$ on $H$ defined by

$$
\alpha(g) x=\pi(g) x+b(g), g \in G, x \in \hbar,
$$

where $\hbar$ is the canonical affine Hilbert space associated with $H^{0}$.

Definition 2.3. A continuous real valued kernel $\Psi$ on a topological space $X$ is conditionally of negative type if $\Psi(x, x)=0, \quad \Psi(x, y)=\Psi(y, x)$, for all $x, y \in X$, and $\sum_{i=1}^{n} \sum_{j=1}^{n} c_{i} c_{j} \Psi\left(x_{i}, x_{j}\right) \leq 0$, for any elements $x_{1}, x_{2}, \cdots, x_{n}$ in $X$, and any real numbers $c_{1}, c_{2}, \cdots, c_{n}$ with $\sum_{i=1}^{n} c_{i}=0$. A continuous real value function $\psi$ on a topological group $G$ is conditionally of negative type if the kernel on $G$ defined by $(g, h) \mapsto \psi\left(h^{-1} g\right)$ is conditionally of negative type.

Example 2.4. Let $G$ be a topological group, and let $\alpha$ be an affine isometric action of $G$ on a real Hilbert space $\hbar$, according to Example C. 2.2 ii in [1], for any $\xi \in \hbar$, the function

$$
\psi: G \rightarrow R, \quad \psi(g)=\|\alpha(g) \xi-\xi\|^{2}
$$

is conditionally of negative type.

In particular, for any orthogonal representation $\pi$ on $\hbar$ and for any $b \in Z^{1}(G, \pi)$, the function $g \mapsto\|b(g)\|^{2}$ is conditionally of negative type. 
Theorem 2.5. [1] Let $\pi$ be an orthogonal representation of the topological group $G$ on a real Hilbert space $\hbar$. Let $b \in Z^{1}(G, \pi)$, with associated affine isometric action $\alpha$. The following statements are equivalent:

1) $b$ is bounded;

2) all the orbits of $\alpha$ are bounded;

3) some orbit of $\alpha$ is bounded;

4) $\alpha$ has a fixed point in $\hbar$.

Definition 2.6. A topological group $G$ has Property $(F H)$ if every affine isometric action of $G$ on a real Hilbert space has a fixed point. Let $H$ be a closed subgroup of $G$. The pair $(G, H)$ has Property $(F H)$ if every affine isometric action of $G$ on a real Hilbert space has an $H$-fixed point.

The following theorem describes connection among bounded functions conditionally of negative type, Property (FH) and cohomology groups pair $(G, H)$.

Theorem 2.7. [1] Let $G$ be a topological group, $H$ be a closed subgroup of $G$. The following statements are equivalent:

1) $\operatorname{Res}_{G}^{H}: H^{1}(G, \pi) \rightarrow H^{1}\left(H,\left.\pi\right|_{H}\right)$ is the zero mapping, for every orthogonal representation $\pi$ of $G$,

2) $\operatorname{pair}(G, H)$ has Property $(\mathrm{FH})$,

3) every function conditionally of negative type on $G$ is H-bounded.

Theorem 2.8. [1] $G$ be a topological group, $H$ be a closed subgroup of $G$.

1) If Pair $(G, H)$ has relative Property (T), then pair $(G, H)$ has Property (FH).

2) If Pair $(G, H)$ has relative Property (T), then every function conditionally of negative type on $G$ is $H$ bounded.

3) Assume that $G$ is a $\sigma$-compact locally compact group and that pair $(G, H)$ has Property (FH), then pair $(G, H)$ has relative Property $(\mathrm{T})$.

Proof. By virtue of [1] Remark 2.12.5: if the pair $(G, H)$ has relative Property (T), then $(G, H)$ has Property (FH) and Theorem 2.7 1), 2) is obvious and 3) is the Delorme-Guichardet Theorem applied to the pair $(G, H)$, consisting of a group $G$ and a subgroup $H$ (see [1], Exercise 2.14.9).

Theorem 2.9. [4] Let $G$ be a topological group and $H_{1}, H_{2}$ be subgroups of $G$ such that $G$ is generated by $H_{1} \cup H_{2}$. Each pair $\left(G, H_{i}\right)$ has relative Property $(T)$. Then $G$ has Property $(T)$.

\section{Relative Property (T) of Pairs for Topological Groups and Subsets}

When $G$ be a locally compact group, Cornulier extended the definition of relative Property (T) to pairs $(G, X)$, where $X$ is any subset of $G$, and then established various characterizations of relative Property (T) for the pair $(G, X)$, which were already known in [1] [9] for the case that $G$ is a topological group, $H$ is a closed subgroup. We extend the definition of relative Property (T) to pairs $(G, X)$, where $G$ is a topological group, $X$ is any subset of $G$, and then established similar characterizations of relative Property (T) for the pair $(G, X)$.

Definition 3.1. Let $G$ be a topological group, and $X$ is any subset of $G$. The pair $(G, X)$ has relative Property $(T)$ if there exist a compact subset $Q$ and a real number $\varepsilon>0$ such that, whenever $\pi$ is $a$ continuous unitary representation of $G$ on a Hilbert space $\hbar$ for which there exists a vector $\xi \in \hbar$ of norm 1 with $\sup _{q \in Q}\|\pi(q) \xi-\xi\|<\varepsilon$, then there exists an invariant vector, namely a vector $\eta \neq 0$ in $\hbar$ such that $\pi(g) \eta=\eta$ for all $g \in X$.

Definition 3.2. Let $G$ be a topological group, and $X$ is any subset of $G$. The pair $(G, X)$ has Property $(F H)$ if every affine isometric action of $G$ on a real Hilbert space has an $X$-fixed point.

The following theorem will establish some characterizations of relative Property (T) for a pair $(G, X)$.

Theorem 3.3. Let $G$ be a topological group and $X$ be any subset of $G$.

1) If pair $(G, X)$ has relative Property (T), then pair $(G, X)$ has Property (FH).

2) If pair $(G, X)$ has relative Property (T), every function conditionally of negative type on $G$ is $X$ bounded.

3) Assume that $G$ is a $\sigma$-compact locally compact group and that pair ( $G, X)$ has Property (FH), then the pair $(G, X)$ has relative Property (T).

We will apply the following lemma to prove above theorem:

Lemma 3.4. Let $G$ be a topological group, $X$ be any subset and $H$ be a close subgroup of $G$, where 
$X$ generates $H$. Then the pair $(G, H)$ has relative Property $(T)$ if and only if the pair $(G, X)$ has relative Property (T).

Proof. Necessity. Since the pair $(G, X)$ has relative Property (T), there exist a compact subset $Q$ and a real number $\varepsilon>0$ such that whenever $\pi$ is a continuous unitary representation of $G$ on a Hilbert space $\hbar$ for which there exists a vector $\xi \in \hbar$ of norm 1 with $\sup _{q \in Q}\|\pi(q) \xi-\xi\|<\varepsilon$, then there exists an invariant vector $\eta \neq 0$ in $\hbar$ such that $\pi(g) \eta=\eta$ for all $g \in X$. Hence

1) $\pi\left(g^{2}\right) \eta=\pi(g) \pi(g) \eta=\pi(g) \eta=\eta$.

2) Since $\pi(g) \eta=\eta, \pi\left(g^{-1}\right) \pi(g) \eta=\pi\left(g^{-1}\right) \eta=\eta$.

3) $\pi\left(g_{1} g_{2}\right) \eta=\pi\left(g_{1}\right) \pi\left(g_{2}\right) \eta=\pi\left(g_{1}\right) \eta=\eta$.

4) According to $\pi$ is a continuous unitary representation of $G$ on a Hilbert space $\hbar$, if $g_{n} \rightarrow g$, where $g_{n} \in X$, then $\pi\left(g_{n}\right) \eta \rightarrow \pi(g) \eta$.

According to 1$), 2), 3)$ and 4$)$, pair $(G, H)$ has relative Property (T).

Sufficiency. It follows from definition of relative Property $(\mathrm{T})$.

Proof of Theorem 3.3. According to Lemma 3.4, the pair $(G, H)$ has relative Property (T), where $X$ generate $H$, then by Theorem 2.8, every function conditionally of negative type on $G$ is H-bounded.

1) By Theorem 2.7, the pair $(G, H)$ has Property (FH), hence the pair $(G, X)$ has Property (FH).

2) Since every function conditionally of negative type on $G$ is $H$-bounded, it is $X$-bounded too.

3) According to [3] (Theorem 1.1), the result holds.

Example 3.5 Let $K$ be a any non-archimedean local field, we consider the following subgroups of $S L_{2}(K)$ :

$$
\begin{aligned}
& N_{1}=\left\{\left(\begin{array}{ll}
1 & x \\
0 & 1
\end{array}\right): x \in K\right\} \\
& N_{2}=\left\{\left(\begin{array}{ll}
1 & 0 \\
x & 1
\end{array}\right): x \in K\right\}
\end{aligned}
$$

Then the pair $\left(S L_{2}(K), N_{i}\right), i=1,2,\left(S L_{2}(K), N_{1} \cup N_{2}\right)$ do not have relative Property (T).

In fact, if $\left(S L_{2}(K), N_{1}\right)$ has relative Property $(\mathrm{T})$, then $\left(S L_{2}(K), N_{2}\right)$ has relative Property. Since the group $S L_{2}(K)$ is generated by $N_{1} \cup N_{2}$, by Theorem 2.9, $S L_{2}(K)$ has Property (T). This contradicts the fact that $S L_{2}(K)$ does not have Property (T). By Lemma 3.4, $\left(S L_{2}(K), N_{1} \cup N_{2}\right)$ do not have relative Property (T).

Remark 3.6. By Theorem 3.3, $S L_{2}(K)$ does not have Property $(F H) .\left(S L_{2}(K), N_{1} \cup N_{2}\right)$ do not have relative Property (FH). According to [1] (remark: 1.4 .4 (iii) on Page 47), groups $N_{i}, i=1,2$ do not have Property (T), hence do not have Property (FH).

Theorem 3.7. Let $G$ be a topological group, $X_{1}, X_{2}, \cdots, X_{n}$ be subsets. Denote by $X_{1} \cdots X_{n}$ the pointwise product $\left\{x_{1} \cdots x_{n} \mid\left(x_{1}, \cdots, x_{n}\right) \in X_{1} \times X_{2} \times \cdots \times X_{n}\right\}$. Suppose that, for every $i,\left(G, X_{i}\right)$ has relative Property (T). Then pair $\left(G, X_{1} \cdots X_{n}\right)$ has Property $(F H)$.

Proof. It suffices to prove the case when $n=2$, since the result follows by induction on $n$. By Theorem 3.3, $\left(G, X_{i}\right)$ has Property (FH), and every function conditionally of negative type on $G$ is $X_{i}$-bounded. Let $\psi$ be any conditionally negative definite function on G. According to the inequality, $\psi(g h)^{\frac{1}{2}} \leq \psi(g)^{\frac{1}{2}}+\psi(h)^{\frac{1}{2}}$, the pair $\left(G, X_{1} \cdot X_{2}\right)$ is $X_{1} \cdot X_{2}$-bounded. Hence, pair $\left(G, X_{1} \cdots X_{n}\right)$ is $X_{1} \cdots X_{n}$-bounded.

Remark 3.8. In [3], Cornulier show when $G$ be a locally compact group for above theorem, then pair $\left(G, X_{1} \cdots X_{n}\right)$ has relative Property $(T)$.

Theorem 3.9. Let $G$ be a topological group, $X_{1}, X_{2}, \cdots, X_{n}$ be subsets. Suppose that, for every $i$, $\left(G, X_{i}\right)$ has relative Property ( $T$ ), then we have:

1) the pair $\left(G, X_{1} \cup \cdots \cup X_{n}\right)$ has Property (FH).

2) if $X_{1} \cap \cdots \cap X_{n} \neq \phi$, then the pair $\left(G, X_{1} \cap \cdots \cap X_{n}\right)$ has Property (FH).

Proof. 1) $\forall g \in \bigcup_{i=1}^{n} X_{i}$, then there exists a $X_{i}$ such that $g \in X_{i}$. Since pair $\left(G, X_{i}\right)$ has relative Property (T), let $\psi$ be any function conditionally of negative type on $G$, then $\psi(g)<\infty$, that is, $\psi$ is an $\bigcup_{i=1}^{n} X_{i}$ bounded function. Similarly, we can easily prove result 2). 
Theorem 3.10. Let $G$ be a topological group.

1) Let $H$ be a subgroup of $G, X_{1} \subset X_{2} \subset H$. If the pair $\left(H, X_{2}\right)$ has relative Property (T), then $\left(G, X_{1}\right)$ has relative Property (T).

2) Let $H$ be a subgroup of $G, X_{1} \subset X_{2} \subset H$. If the pair $\left(H, X_{2}\right)$ has Property (FH), then $\left(G, X_{1}\right)$ has Property (FH).

3) Let $G_{1}, \cdots, G_{n}$ be subgroups of $G, \quad X_{i} \subseteq G_{i}, i=1, \cdots, n$. Let $H$ be a topological group generated by $G_{1} \cup \cdots \cup G_{n}$. If $\left(G_{i}, X_{i}\right)$ has relative Property (T) for every $i$, then the pair $\left(G, \bigcup_{i=1}^{n} X_{i}\right)$ has Property (FH).

Proof. 1) Since $\left(H, X_{2}\right)$ has relative Property (T), if there exist a compact subset $Q$ and a real number $\varepsilon>0$ such that, $\pi$ is a continuous unitary representation of $G$ on a Hilbert space $\hbar$, then $\pi$ is a continuous unitary representation of $H$ on a Hilbert space $\hbar$ for which there exists a vector $\xi \in \hbar$ of norm 1 with $\sup _{q \in Q}\|\pi(q) \xi-\xi\|<\varepsilon$, there exists an invariant vector, namely a vector $\eta \neq 0$ in $\hbar$ such that $\pi(g) \eta=\eta$ for all $g \in X_{2}$. According to $X_{1} \subset X_{2}, \pi(g) \eta=\eta$ for all $g \in X_{1}$. Hence $\left(G, X_{1}\right)$ has relative Property (T).

2) Since $\left(H, X_{2}\right)$ has Property (FH), every affine isometric action of $G$ on a real Hilbert space is an affine isometric action of $H$, and thus it has an $X_{2}$-fixed point. Hence, it has an $X_{1}$-fixed point. That is, $\left(G, X_{1}\right)$ has Property (FH).

3) It suffices to prove the case when $n=2$, since then the result follows by induction. According to 1$)$ and Theorem 3.9, $X_{1} \subseteq X_{1} \cup X_{2} \subseteq H \subseteq G$, for every $i$, the pair $\left(H, X_{i}\right)$ has relative Property (T), then the pair $\left(H, X_{1} \cup X_{2}\right)$ has Property (FH). By using the result in 2), the pair $\left(G, X_{i} \cap X_{2}\right)$ has Property (FH).

In [4], M.Ershov and A. Jaikin-Zapirain showed the following facts: Suppose $G$ be a group generated by subgroups $H_{1}, \cdots, H_{n}$, and the pair $\left(G, H_{i}\right)$ has relative Property (T) for each $i$, and any two subgroups $H_{i}$ and $H_{j}$ are $\varepsilon$-orthogonal for some $\varepsilon<\frac{1}{n-1}$. Then $G$ has Property (T).

We will naturally ask: Can we remove the hypothesis "any two subgroups $H_{i}$ and $H_{j}$ are $\varepsilon$-orthogonal for some $\varepsilon<\frac{1}{n-1}$ ”?

The following Theorem solves the above problem partially:

Theorem 3.11. Let $G$ be a $\sigma$-compact locally compact group generated by subgroups $H_{1}, \cdots, H_{n}$. Suppose that the pair $\left(G, H_{i}\right)$ has relative Property $(T)$ for each $i$. Then $G$ has Property $(T)$.

Proof. According to Theorem 3.9, the pair $\left(G, H_{1} \cup \cdots \cup H_{n}\right)$ has Property (FH). Then by Theorem 3.3, the pair $\left(G, H_{1} \cup \cdots \cup H_{n}\right)$ has relative Property (T). Since $G$ is a group generated by subsets $H_{1}, \cdots, H_{n}$, according to Lemma 3.4, the pair $(G, G)$ has relative Property (T), then $G$ has Property (T).

\section{Acknowledgements}

The work is finished during the first author visiting Auburn University. He thanks Professor Tin-Yau Tam for his invitation to visit Auburn University and participate the Linear Algebra Seminar.

\section{References}

[1] Kekka, B., Harpe, P.D.L. and Valette, A. (2008) Kazhdan’s Property T. Cambridge University Press, Cambridge.

[2] Chifan, I. and Ioana, A. (2011) On Relative Property (T) and Haagerup's Property. Transactions of the American Mathematical Society, 363, C6407-C6420. http://dx.doi.org/10.1090/S0002-9947-2011-05259-1

[3] Cornulier, Y. (2006) Relative Kazhdan Property. Annales Scientifiques de1'École Hormale Supérieure, 39, $301-333$.

[4] Ershov, M. and Jaikin-Zapirain, A. (2010) Property (T) for Noncommutative Universal Lattices. Inventiones Mathematicae, 179, 303-347. http://dx.doi.org/10.1007/s00222-009-0218-2

[5] Kazhdan, D. (1967) Connection of Dual Space of Group with the Structure of Its Closed Subgroups. Functional Analysis and Its Applications, 1, 63-65. http://dx.doi.org/10.1007/BF01075866

[6] Margulis, G. (1982) Finitely-Additive Invariant Measures on Euclidean Spaces. Ergodic Theory and Dynamical Systems, 2, 383-396. http://dx.doi.org/10.1017/S014338570000167X 
[7] Harpe, P.D.L., Valette, A. and Marc, B. (1989) La Propriété (T) de Kazhdan pour les groups localement compacts. Astérisque 175, Société mathématique de France (SMF).

[8] Shalom, Y. (1999) Bounded Generation and Kazhdan’s Property (T). Publications Mathématiques de l'Institut des Hautes Études Scientifiques, 90, 145-168. http://dx.doi.org/10.1007/BF02698832

[9] Jolissaint, P. (2005) On Property (T) for Pairs of Topological Groups. L’Enseignement Mathématique, 51, 31-45. 
Scientific Research Publishing (SCIRP) is one of the largest Open Access journal publishers. It is currently publishing more than 200 open access, online, peer-reviewed journals covering a wide range of academic disciplines. SCIRP serves the worldwide academic communities and contributes to the progress and application of science with its publication.

Other selected journals from SCIRP are listed as below. Submit your manuscript to us via either submit@scirp.org or Online Submission Portal.
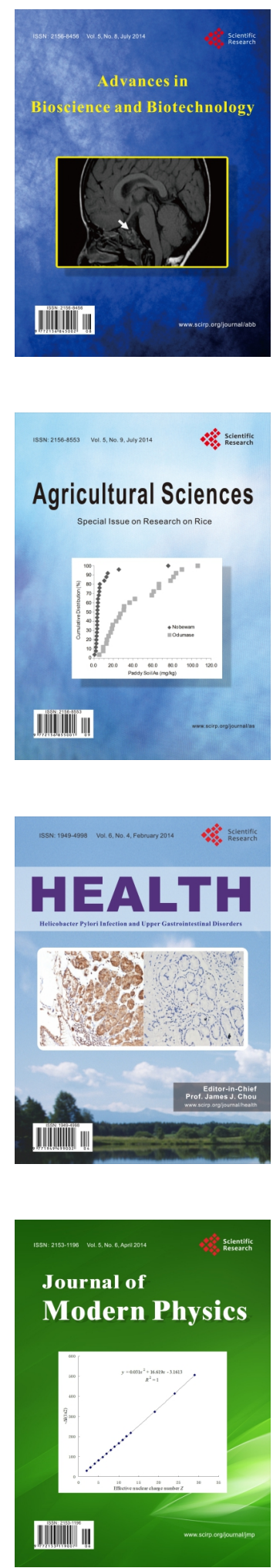
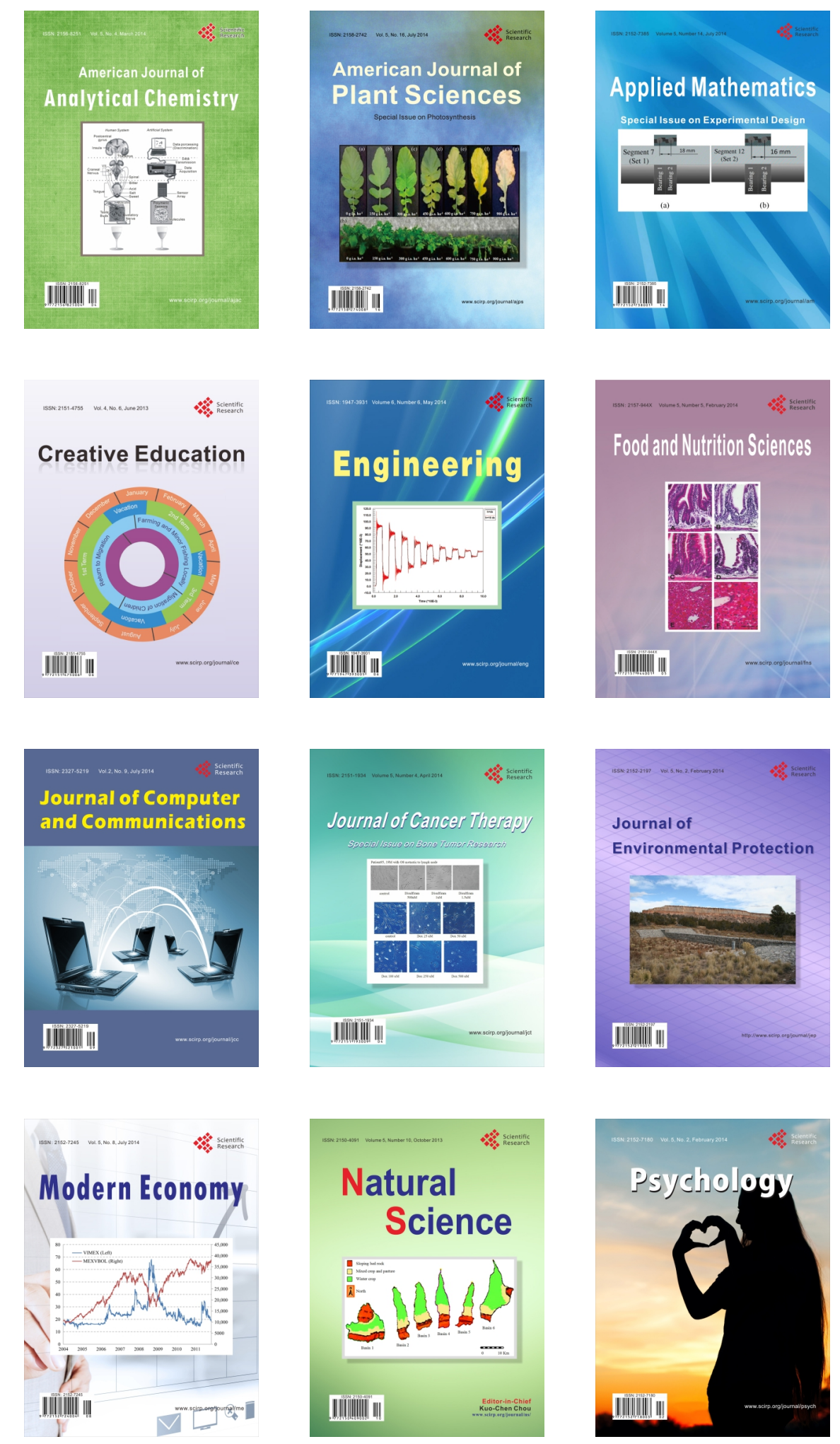